\title{
Cultural factors that affect sexual and reproductive health in Malawi
}

\section{Thomas Bisika}

\section{Background}

Malawi is one of the countries worst hit by the HIV/AIDS epidemic, with an infection rate among the childbearing age group of $12.0 \% .^{1}$ Young people aged $15-24$ years account for $46 \%$ of new HIV infections, of which $60 \%$ occur in girls. HIV prevalence is even higher in some rural districts $(17.5 \%, p<0.01) .^{2}$ In common with many other sub-Saharan Africa countries, HIV in Malawi is mainly spread through heterosexual sex, hence it is an important reproductive health issue. ${ }^{3}$ HIV/AIDS control efforts are hampered by low voluntary counselling and testing uptake, ${ }^{4}$ which indicates that a high level of HIV/AIDS knowledge is not translating into significant behaviour change.

The maternal mortality rate almost doubled in Malawi between $1992^{5}$ and $2000^{6}$ and still remains very high. Furthermore, there are many other sexual and reproductive health (SRH) problems, which include unwanted pregnancies, reproductive tract infections including sexually transmitted infections (STIs), abortion complications, sexual and gender-based violence, infertility, and adolescent reproductive health.

The role of condom use for dual protection has not yet been fully taken advantage of in Malawi ${ }^{7}$ and, like many African countries, Malawi needs to reposition family planning through the provision of comprehensive SRH services for both men and women that embrace and invigorate family planning. ${ }^{8}$ Poverty explains much of the gender dimension of the HIV epidemic in Malawi. Research has already concluded that improving economic opportunities for women may reduce their need to rely on men who pay them for sex. ${ }^{9}$

\section{HIV/AIDS knowledge, attitudes, practices and behaviour}

Despite a high level of HIV awareness, which is almost universal in Malawi, communities experience many SRH health problems that include HIV/AIDS, STIs and unwanted pregnancies, especially in teenagers. This perhaps means that there is a need to distinguish HIV awareness from actual HIV/AIDS knowledge. Very few people are able to differentiate HIV from AIDS. Further HIV/AIDS education through the existing communication channels such as radio, friends, schools, health talks, newspapers and posters is thus required. People know a number of ways in which HIV can be transmitted (e.g. unprotected sexual intercourse, sharing razor blades and use of unsterilised needles). Many people in Malawi still identify high-risk HIV groups as people with multiple partners, conscripts and commercial sex workers. This creates a degree of false security in some individuals who are not members of these high-risk groups.

J Fam Plann Reprod Health Care 2008; 34(2): 79-80

Department of Social Affairs, African Union Commission, Addis Ababa, Ethiopia

Thomas Bisika, ScD, Head of Health and Population (also Research Fellow, Centre for Social Research, University of Malawi, Zomba, Malawi)

Correspondence to: Dr Thomas Bisika, Department of Social Affairs, African Union Commission, PO Box 3243, Addis Ababa, Ethiopia. E-mail: BisikaT@africa-union.org
Mother-to-child transmission of HIV/AIDS is not that well known, suggesting that this area has not yet been emphasised in HIV/AIDS education. This adversely affects the rapid scaling up of mother-to-child transmission prevention programmes.

\section{Cultural practices that predispose people to HIVIAIDS and other STIs}

A larger proportion of Malawians consider themselves at risk of getting HIV/AIDS as compared to those who think that anybody can get HIV/AIDS. Many people acknowledge that there are cultural practices and beliefs that predispose people to HIV/AIDS in Malawi. These practices and beliefs include chokolo (wife inheritance), fisi (a man who is brought to have sex with young women during an initiation ceremony), chinamwali (initiation ceremonies) and kuchotsa fumbi (forced sex).

Lack of parental involvement and/or guidance in partner selection for those intending to marry, jando (an initiation ceremony for adolescent boys where one razor blade could be used for circumcising a number of participants), manganje (a cultural dance that can provoke sexual desire) and siyala (an overnight dance performed to mark religious events for Moslems but which may result in immoral behaviour) also place people at risk of HIV. Early marriage, which is often associated with older men marrying younger women, is also a risky cultural practice.

In some cultures people are also supposed to abstain from sex when the woman is breastfeeding or has just given birth so that the milk remains 'cold'. The milk is considered 'hot' once sex is resumed. 'Cold' milk is very important and is sometimes used in cleaning the infant's eyes when they become infected. ${ }^{10}$

\section{Social and cultural practices that prevent behaviour change}

In the absence of a cure, prevention becomes the only weapon in the fight against HIV. Behaviour change is very important in HIV prevention. Many people in Malawi report that they have changed their behaviour after hearing of HIV/AIDS; however, cultural practices that prevent people from changing their behaviour are mitala (polygamy), chokolo, chinamwali and early marriage.

\section{Sexual behaviour and condom use}

Condoms are important in the avoidance of pregnancy, STIs and HIV. This dual protection aspect of the condom seems not to have been fully utilised in Malawi. Only about one-quarter of those individuals having sex report that they have used a condom. Cultural practices and beliefs that can prevent condom use exist in many communities and these include religion and unfaithfulness. Some people also think that one cannot enjoy sex when using a condom ("you do not eat a sweet while it is still in its cover") while others believe that condoms cause zilonda (sores).

\section{Premarital and extramarital sex}

Premarital and extramarital sex are important drivers of the AIDS epidemic in Malawi. However, there are cultural practices and beliefs that can reduce premarital sex. These include "being afraid of making someone pregnant as this can prove that one was engaged in sex outside marriage", 
virginity requirement at marriage, the belief that girls have razors in their vagina, religion, chitomero (a practice whereby a girl is allocated a partner in advance), and in some communities if a girl is found pregnant the mother pays a chicken to the chief. In some cultures a man is not supposed to have extramarital sex when his wife is pregnant for fear that she will give birth to a weak or deformed baby. In many areas there is still high stigmatisation of STIs including HIV/AIDS, which may discourage premarital and extramarital sex.

Conversely, there are cultural practices and beliefs that promote extramarital sex such as postpartum abstinence, which can lead a man to seek sex elsewhere. Male circumcision can also have unintended consequences in creating false security among those circumcised. Other problems are lack of respect for women, lobola (where some men believe that they have paid for the wife), wife inheritance and polygamy.

\section{Family planning and contraception}

With respect to family planning and contraception, a number of methods are used in Malawi including injections, pills and condoms. Family planning knowledge is generally higher among women, especially older individuals. However, many people have never used any contraceptive method. Many women have tried the injection most likely because they have some degree of control over this method and it is convenient because it is not taken on a daily basis. This method can also be used without the knowledge of the male partner.

Cultural practices and beliefs that can prevent people from avoiding pregnancy exist in many communities. These include religion (i.e. "be fruitful and multiply"), relying on condoms (which may be used incorrectly at times), mkuzi or chingwe (a rope that is traditionally believed to protect a women from becoming pregnant) and kuchotsa fumbi, which precludes the use of contraception. Misconceptions about family planning (e.g. some women think that they will become permanently infertile), religion, love for dry sex and male opposition also prevent some people from avoiding pregnancy. In many communities the belief is that one should not have sex during menstruation. Since some contraceptive methods may result in prolonged periods of bleeding, some women may decline to use such methods for fear of keeping their husbands waiting for longer periods, which may lead some men to seek sex elsewhere.

\section{Conclusions}

Cultural practices, religion and beliefs combine to put men, women, boys and girls at risk of HIV/AIDS, STIs and unwanted pregnancy, and may prevent people from adopting protective behaviours. The dual protection aspect of the condom has not been taken advantage of in many communities and in general is not a popular family planning method.
One cultural practice can predispose a person to many SRH risks (e.g. fisi and chokolo can increase the risk of STIs, HIV/AIDS and pregnancy). It is also interesting that many people believe that there are more barriers to avoiding HIV/AIDS and using condoms than simply changing attitudes. This suggests that cultural factors have a much stronger influence on actual practices than on attitudes. This is worrying because it is the actual practices that predispose people to STIs, HIV/AIDS and pregnancy. A significant behaviour change would translate to positive SRH practices, but in the case of Malawi this has not yet happened. There is, therefore, a great need to intensify behaviour change communication and curtail the social and cultural factors that predispose men, women, boys and girls to HIV/AIDS, STIs and unwanted pregnancy.

Statements on funding and competing interests

Funding None identified.

Competing interests None identified.

\section{Author's note}

This paper is based on the results of the UNFPA-funded Baseline Survey for the Community-based Sexual and Reproductive Health and Gender Education Programme (COSHAP) of the Malawi Government.

\section{References}

1 Malawi HIV and AIDS Monitoring and Evaluation Report. http://data.unaids.org/pub/Report/2008/malawi_2008_country_ progress_report_en.pdf [Accessed 18 February 2008].

2 Hoffman IF, Tsui A, de Graft-Johnson J, Bisika T, Feluzi H, Kazembe PN. Reducing the STI Burden in Rural Malawi: STD Screening, Case and Contact Treatment, and Condom Promotion. Chapel Hill, NC: University of North Carolina, 2003.

3 Barden-O'Fallon J, deGraft-Johnson J, Bisika T, Benson A, Tsui A. Factors associated with HIV/AIDS knowledge and risk perception in rural Malawi. AIDS Behav 2004; 8: 131-140.

4 deGraft-Johnson J, Paz-Soldan V, Kasote A, Tsui A. 2005. HIV voluntary counseling and testing service preferences in a rural Malawi population. AIDS Behav 2005; 9: 475-484.

5 Malawi Demographic and Health Survey 1992. Claverton, MD: National Statistical Office, Zomba, Malawi and ORC Macro, 1994.

6 Malawi Demographic and Health Survey 2000. Claverton, MD: National Statistical Office, Zomba, Malawi and ORC Macro, 2001.

7 Centre for Social Research, Save the Children Federation USA, Malawi Ministry Health and Population, MEASURE Evaluation. Avoiding Unwanted Pregnancy and Sexually Transmitted Infectionss: A Rural Malawi District Study. Chapel Hill. NC: MEASURE Evaluation, 2004.

8 Bisika T, Short M, Wontumi G-M, Truong J, Tsui A. Repositioning family planning to reduce unmet need. African Renaissance 2007; 4(3\&4): 83-101.

9 Paz-Soldan VA, Bisika T, Benson A, Barden-O'Fallon J, de Graft-Johnson J. Social, economic and demographic determinants of sexual risk behaviors among men in rural Malawi. Afr J Reprod Health 2007; 11(2): 33-46.

10 Bisika T. An Exploratory Study of Traditional Eye Medicine in Malawi. Zomba, Malawi: Centre for Social Research, 2000. 